\title{
New Model Systems to Illuminate Thyroid Organogenesis. Part I: An Update on the Zebrafish Toolbox
}

\author{
Robert Opitz Francesco Antonica Sabine Costagliola
}

Institute of Interdisciplinary Research in Molecular Human Biology, Université Libre de Bruxelles, Brussels, Belgium

\author{
Key Words \\ Thyroid · Development · Zebrafish · Embryo · Transgenesis · \\ Mutagenesis · Live imaging
}

\begin{abstract}
Thyroid dysgenesis (TD) resulting from defects during embryonic thyroid development represents a major cause of congenital hypothyroidism. The pathogenetic mechanisms of TD in human newborns, however, are still poorly understood and disease-causing genetic variants have been identified in only a small percentage of TD cases. This limited understanding of the pathogenesis of TD is partly due to a lack of knowledge on how intrinsic factors and extrinsic signalling cues orchestrate the differentiation of thyroid follicular cells and the morphogenesis of thyroid tissue. Recently, embryonic stem cells and zebrafish embryos emerged as novel model systems that allow for innovative experimental approaches in order to decipher cellular and molecular mechanisms of thyroid development and to unravel pathogenic mechanisms of TD. Zebrafish embryos offer several salient properties for studies on thyroid organogenesis including rapid and external development, optical transparency, ease of breeding, relative short generation time and amenability for genome editing. In this review, we will highlight recent advances in the zebrafish toolkit to visualize cellular dynamics of organ development and discuss specific prospects of the zebrafish model for studies on vertebrate thyroid development and human congenital thyroid diseases.

(c) 2013 European Thyroid Association Published by S. Karger AG, Basel
\end{abstract}

\section{Introduction}

Congenital hypothyroidism $(\mathrm{CH})$ represents the most common congenital endocrine disorder in humans affecting approximately 1 of 2,000-4,000 live births $[1,2]$. The majority of $\mathrm{CH}$ cases are due to thyroid dysgenesis (TD), which might manifest as either athyreosis, thyroid ectopy, hypoplasia or hemiagenesis $[3,4]$. All these phenotypes are considered to result from defects in embryonic thyroid development. The molecular mechanisms underlying TD in human newborns, however, remain poorly understood to date $[2,5]$. This is, in part, due to our still limited understanding on how the function of intrinsic factors (e.g. thyroid-specific transcription factors) and extrinsic signaling cues (e.g. morphogens and growth factors) are integrated to regulate normal thyroid organogenesis [6, 7]. Thyroid morphogenesis is a complex process comprising endodermal precursor cell specification, thyroid bud formation and evagination from ventral foregut endoderm, relocalization of the thyroid primordium to a position distant from its site of origin, functional differentiation of thyroid follicular cells (TFC) and the formation of functionally mature thyroid follicles. Most of our current knowledge on thyroid morphogenesis is derived from examination of mouse embryos $[3,7]$. It was particularly the generation and phenotypic analysis of murine knock-out models for key factors controlling thyroid development (e.g. NKX2.1, PAX8, FOXE1, HEX) that laid the foundation for current models of TFC differentiation and thyroid

Sabine Costagliola

Institute of Interdisciplinary Research in Molecular Human Biology Université Libre de Bruxelles BE-1070 Brussels (Belgium) E-Mail scostag@ulb.ac.be 
organogenesis [8-11]. Despite these critical findings in mice, mutations of the orthologous human genes have been detected in only a minority of TD patients, suggesting that unknown genetic and epigenetic factors might be important for thyroid development $[2,12]$. Thus, probing the causative molecular mechanisms for TD remains a challenging task. In addition to defective gene expression in TFC or their endodermal precursor cells, impaired physical and/or molecular interactions between the developing thyroid primordium and adjacent tissues need to be considered to account for TD. Evidence corroborating the importance of tissue-tissue interactions for proper thyroid development has recently been provided by studies on mouse and zebrafish thyroid development [13-15]. However, very little is known about the identity and source of extrinsic signaling cues influencing thyroid morphogenesis $[6,7]$.

Innovative experimental approaches using novel model systems are therefore required to enhance our understanding of the cellular and molecular mechanisms of thyroid development. One such promising model system is the zebrafish embryo. Over the past two decades, the zebrafish embryo has gained a growing attention as a versatile in vivo model to study basic mechanisms of vertebrate organogenesis [16-18]. More recently, along with an expanding molecular and genetic toolkit, zebrafish has also proven a powerful organism to delineate pathological mechanisms of human diseases at the molecular and cellular level [19-21]. In this review, we first highlight current advances in the zebrafish toolkit to visualize cellular dynamics of organ development and describe how novel transgenic tools can be harnessed to fill gaps in our understanding of the cellular and molecular processes involved in thyroid morphogenesis. We then proceed to discuss the potential impact of recent technological developments on future contributions of genetic zebrafish models to the study of human congenital thyroid diseases.

\section{Salient Features of a Zebrafish Model of Thyroid Development}

Zebrafish represents an evolutionary distant model organism differing in some aspects of its basic thyroid tissue architecture from mammalian species. However, the molecular mechanisms of thyroid organogenesis appear well conserved between zebrafish and mammalian models [22-27]. The thyroid anlage of zebrafish embryos can be detected first around $24 \mathrm{~h}$ post-fertilization (hpf) as a stripe of endodermal cells co-expressing the three tran- scription factors $n k x 2.1 a$, pax $2 a$ and hhex [22, 24]. Along with the formation of the foregut proper, the early anlage transforms into a thyroid bud that begins to evaginate from the ventral floor of the foregut about $8 \mathrm{~h}$ after thyroid anlage specification. Budding of the thyroid primordium from the pharyngeal epithelium is completed at 48 $\mathrm{hpf}$ and is followed by a relocation of the thyroid primordium deeper into the subpharyngeal mesenchyme [15]. Formation of a first follicle immunoreactive for thyroglobulin can be observed around $55 \mathrm{hpf}$. Between 55 and $60 \mathrm{hpf}$, the thyroid primordium starts to expand along the anterior-posterior axis generating dispersed patches of thyroid tissue and thyroidal $\mathrm{T}_{4}$ synthesis becomes detectable between 70 and $80 \mathrm{hpf}$. It should be stressed that the time elapsed between specification of the thyroid anlage in zebrafish embryos and the formation of functionally mature thyroid follicles is only about $48 \mathrm{~h}$. From this brief summary of the timeline of zebrafish thyroid development, it becomes clear that the rapidity of overall zebrafish development holds also true for thyroid morphogenesis. What differs between zebrafish and mouse is the relative timing of some morphogenetic events and the gross anatomy of adult thyroid tissue $[23,26]$. Whereas thyroid follicles are encapsulated in a compact organ in mouse, thyroid follicles are found loosely dispersed along the pharyngeal midline in zebrafish. For a more comprehensive analysis of zebrafish thyroid development in comparison to mammalian systems, the readers are referred to two excellent reviews that recently examined this topic in more detail $[27,28]$.

A zebrafish model of thyroid development offers several advantageous properties that could help to overcome some of the technical limitations inherent to classical mammalian models and should therefore serve as a powerful complementary system to the existing toolbox for studies on thyroid development. A first step in order to acknowledge the potential value of the zebrafish model is to recapitulate those properties that are complementary to mammalian experimental systems. Most importantly, and in contrast to the intrauterine development of mammalian embryos, zebrafish embryos and larvae develop externally making them accessible to experimental manipulations and real-time observations throughout their embryonic and larval development. Another important advantage is that a single healthy female founder fish can lay hundreds of eggs during a single breeding event so that a large number of specimens are available for any given type of analysis. Breeding a cohort of sexually mature fish allows one to readily collect thousands of fertilized eggs at a given time point, enabling the conduct of larger-scale 
experiments with developmentally synchronized embryos. The optical transparency of embryonic and early larval stages permits real-time visualization of developmental processes and their pathological deviations in live embryos. While live monitoring of embryo development was initially limited to grossly detectable tissues and organs (e.g. brain, eyes, skin, heart), the availability of transgenic embryos expressing fluorescent reporter proteins in specific cell types nowadays permits visualization of even small groups of cells deep inside the embryo by means of fluorescent microscopy. Although mouse embryos can be cultured for short periods ex vivo, their opacity in the organogenesis and late gestational phases of development still represents a major technical barrier for live imaging of organ development including thyroid morphogenesis. Collectively, these favorable properties make zebrafish embryos an ideal model to study the cellular and molecular mechanisms of thyroid organogenesis.

\section{The Transgenic Toolkit for Zebrafish Thyroid Organogenesis}

Throughout its development, zebrafish thyroid tissue is of a very small size and is hardly detectable by brightfield microscopy of live embryos. Until recently, the developing thyroid could only be visualized by wholemount in situ hybridization or immunohistochemistry of fixed embryos. One of the key technological developments in zebrafish was the introduction of streamlined protocols and powerful reagents for efficient generation of transgenic embryos [29-32]. The introduction of Tol2 transposon-based transgenesis methods (fig. 1a) together with the development of a suite of gateway-compatible vectors containing Tol2 cis elements greatly enhanced germline transmission frequency of transgenic constructs and spurred the generation of numerous tissue-specific transgenic reporter lines. The confluence of fluorescent reporter line availability, ease of specimen mounting and advanced microscopy technologies enabled unprecedented opportunities for live imaging in order to illuminate the cellular dynamics of tissue and organ morphogenesis [33-35].

Because fluorescent reporter lines allow for innovative experimental approaches to study thyroid development, we recently generated a panel of transgenic zebrafish lines in which the promoter of the zebrafish thyroglobulin ( $\mathrm{tg}$ ) gene was used to drive expression of different fluorescent proteins [15]. The zebrafish $t g$ promoter offers several advantageous properties for the generation of transgenic embryos. Endogenous $t g$ mRNA expression is induced relatively early during thyroid morphogenesis (around 34 hpf) and is confined to thyroid cells. In accordance with the endogenous $t g$ mRNA expression profile, all transgenic embryos generated using the $t g$ promoter display an early onset of reporter gene expression concurrent with formation of the thyroid bud around 35/36 hpf. Moreover, common to all transgenic lines is a strong, thyroidspecific expression of fluorescent proteins.

Although the thyroid develops quite deep inside the embryo, the combination of a strong reporter signal, rapid embryonic development and the transparency of embryonic tissues facilitates time-lapse live imaging and real-time visualization of the dynamic changes in size, shape and location of the developing zebrafish thyroid [15]. In combination with novel fluorescence microscopy technologies for volumetric (4D) time-lapse imaging [36], these new transgenic tools hold great promise to develop a dynamic three-dimensional atlas of thyroid morphogenesis in zebrafish embryos. Particularly powerful for this purpose are double transgenic embryos expressing different fluorescent reporters in thyroid cells and adjacent tissues to monitor thyroid organogenesis in relation to surrounding tissues and organs (fig. 1b, c). For example, 4D time-lapse imaging of transgenic embryos expressing the red fluorescent protein mCherry in all thyroid cells and enhanced green fluorescent protein (eGFP) in endothelial cells proved a very sensitive and information-rich imaging approach to identify specific pharyngeal blood vessels guiding thyroid relocalization in zebrafish embryos [15]. The thyroid-specific expression of reporter proteins also enabled for the first time to stain all thyroid cells in fixed embryos by means of immunofluorescence. This allowed us to harness the power of confocal microscopy for optical sectioning of zebrafish embryos and the generation of high-resolution $3 \mathrm{D}$ models of the thyroid and heart outflow tract region in embryos with normal and defective vascular development [15]. Such $3 \mathrm{D}$ modeling proved invaluable for the understanding of the anatomical relationships between outflow tract remodeling and thyroid morphogenesis.

To date, the cellular dynamics of mammalian thyroid organogenesis are poorly understood due to the inaccessibility of embryonic thyroid tissue for live imaging. Zebrafish embryos expressing subcellularly localized fluorescent reporters in TFC offer new and promising tools to study the cellular behavior of TFC in real-time during embryonic development. Thyroid-specific expression of a membrane-tethered version of mCherry facilitates visualization of dynamic changes in cell morphology as well 
a
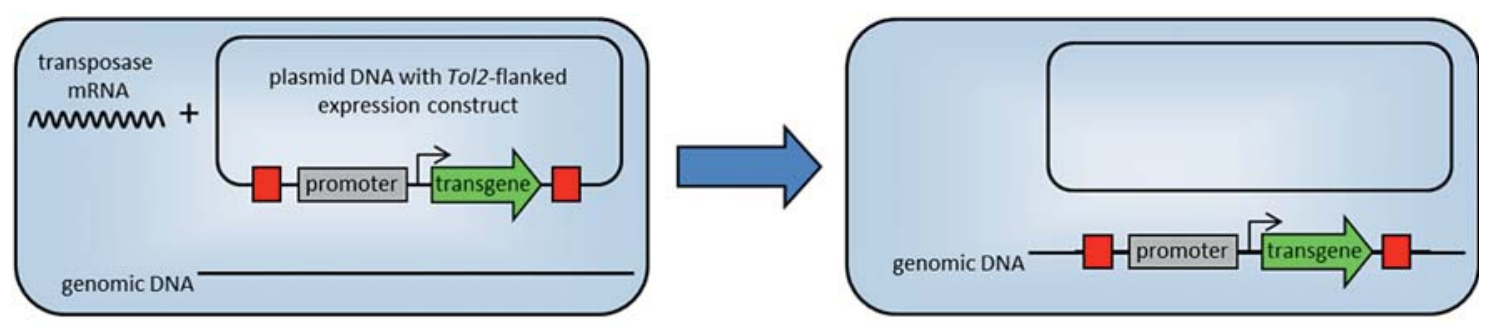

b
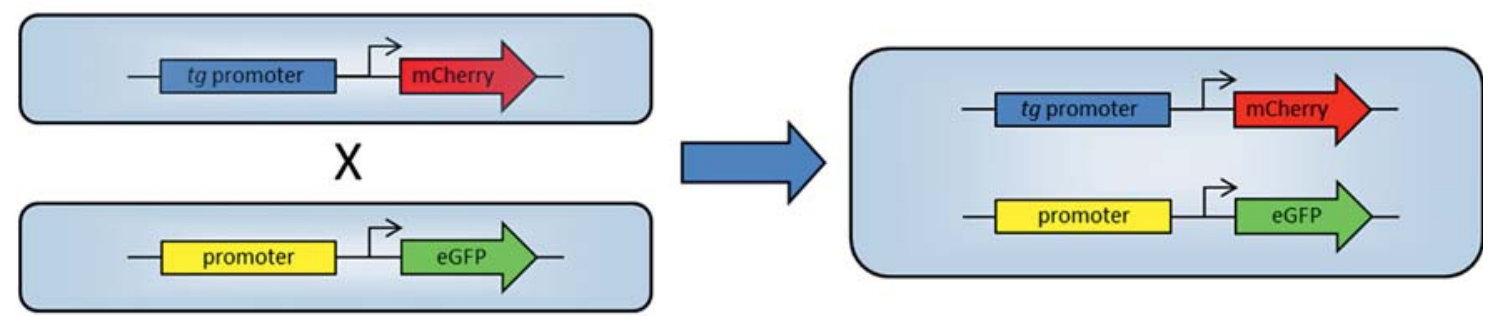

c
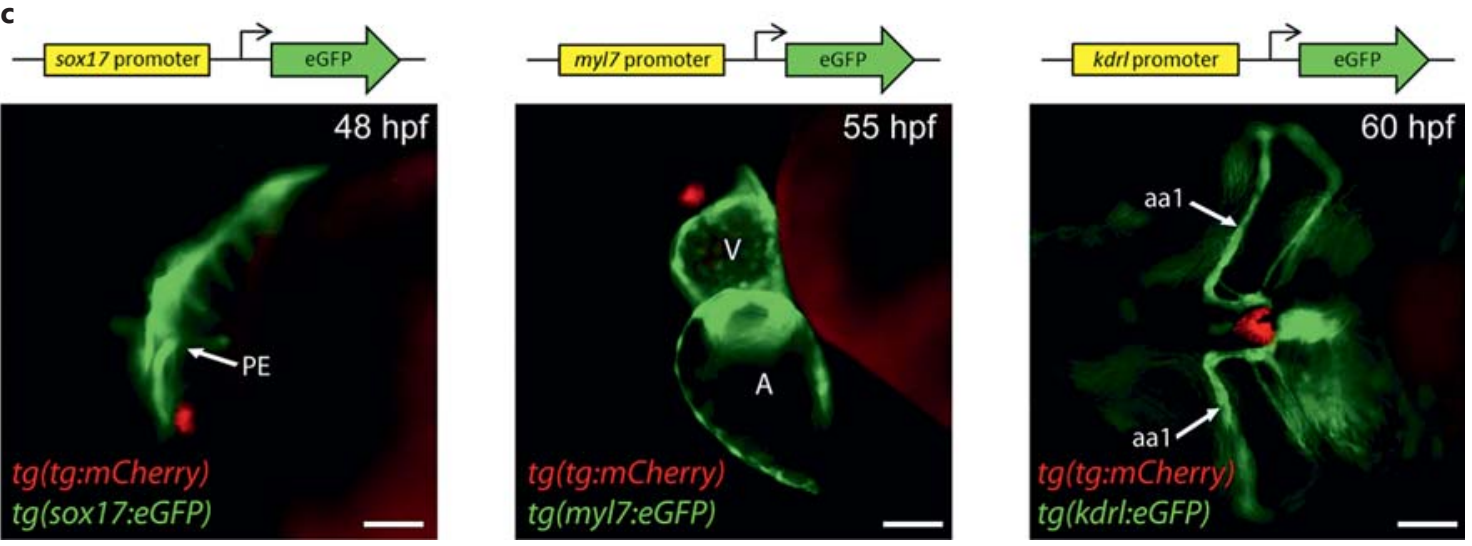

Fig. 1. Generation of transgenic thyroid reporter lines. a Schematic illustration of Tol2 transgenesis. Capped transposase RNA is generated by in vitro transcription and a transposon donor vector containing an expression construct flanked by two Tol2 sites (red blocks) is assembled by gateway cloning. The expression construct used for generation of thyroid reporter lines contains a 3.2$\mathrm{kb}$ region of the zebrafish $\mathrm{tg}$ promoter upstream of the coding sequence of fluorescent reporter proteins [15]. The transposon donor vector is co-injected with transposase mRNA into 1- to 2 -cell stage embryos where the Tol2-flanked expression construct is excised from the donor plasmid by transposase and integrated into the genome of somatic cells (transient transgenesis) or germ cells (stable transgenesis). Major advantages of Tol2 transgenesis are the high efficiency of Tol2 construct insertions and germline transmissions. b Once a stable thyroid reporter line is established, simple crosses of founder fish allow to generate double transgenic embryos expressing a specific fluorescent reporter (e.g. mCherry) in thyroid cells and a different reporter (e.g. eGFP) in adjacent tissues. c Double transgenic embryos are powerful tools for live imaging of thyroid development in relation to surrounding tissues and organs. Live embryos were immobilized in low-melting agar and thyroid development was monitored by epifluorescence microscopy in relation to the morphogenesis of foregut endoderm ( $\operatorname{tg}(\operatorname{sox} 17: e G F P)$, lateral view), myocardium $(\operatorname{tg}(m y l 7: e G F P)$, lateral view), and vascular endothelium ( $\operatorname{tg}(k d r l: e G F P)$, ventral view). All embryos shown are oriented with anterior to the left. $\mathrm{PE}=$ Pharyngeal endoderm; $\mathrm{V}=$ ventricle; $\mathrm{A}=$ atrium; $\mathrm{AA} 1=$ aortic arch artery 1 . Scale bar: $50 \mu \mathrm{m}$. 



Fig. 2. Live imaging of transgenic reporter embryos permits realtime visualization of cellular dynamics. In $\operatorname{tg}(\operatorname{tg}: m$ Cherry-memb) embryos (a-c), apical constriction (arrowheads in a) of follicular cells are prominent facilitating the identification of sites of new follicle formation (arrowhead in c). Note also the dynamic changes in thyroid cell morphologies (arrowhead in $\mathbf{b}$ ) during the period of thyroid expansion along the pharyngeal midline. Live imaging of embryos expressing membrane-targeted mCherry in thyroid cells and eGFP in endothelial cells highlights the direct cell-cell contacts between thyroid cells and the endothelium of the hypobranchial artery (d). Real-time analyses of thyroid cell number is facilitated by live imaging of transgenic embryos expressing nuclear eGFP in thyroid cells $(\mathbf{e}, \mathbf{f})$. In addition, the faint cytoplasmic reporter signal permits visualization of intracellular vesicles dur- ing the process of folliculogenesis (arrowhead in $\mathbf{f}$ ). The global dynamics of thyroid tissue expansion can be nicely visualized in transgenic embryos co-expressing membrane-targeted mCherry and nuclear GFP in thyroid cells $(\mathbf{g})$. Note the compact mass of thyroid cells in the anterior part and the prominent cell extensions (arrowheads in $\mathbf{g}$ ) connecting small clusters of thyroid cells in the posterior part of the expanding thyroid primordium of a 72-hpf embryo. Live imaging of double transgenic $\operatorname{tg}(\mathrm{tg}$ : $m$ Cherry-memb; tg:eGFP-nuc) embryos $(\mathbf{h}-\mathbf{j})$ also offers great promise to visualize in real-time the cellular dynamics of de novo follicle formation (asterisk). All panels show ventral views and anterior is to the left. Confocal sections are shown in $\mathbf{a - f}$ and $\mathbf{h}-\mathbf{j} ; \mathbf{g}$ shows a confocal projection. Scale bar: $10 \mu \mathrm{m}$. as other fine cellular details (fig. 2a-d). Live imaging of $\operatorname{tg}$ (tg:mCherry-memb) embryos, for example, demonstrates highly dynamic changes in size and shape of TFC during thyroid expansion.

Of particular importance for the understanding of organ morphogenesis are insights into the spatiotemporal dynamics of tissue growth and cell proliferation. Analyses of TFC number in live $\operatorname{tg}(\mathrm{tg}: m$ Cherry-memb) embryos can become very challenging due to the complex cell morphologies encountered in the thyroid tissue during the phase of thyroid expansion and folliculogenesis. To facilitate real-time cell counting in live embryos, we recently generated new transgenic lines expressing GFP fusion proteins with a nuclear localization signal specifically in TFC (fig. $2 \mathrm{e}-\mathrm{g}$ ). These new lines not only permit analyses of the kinetics of thyroid growth by the quantification of TFC numbers in live embryos, but also provide a superior sensitivity to detect conditions of mild and moderate thyroid hypoplasia in response to specific experimental treatments [Opitz, Trubiroha and Costagliola, unpubl. data]. In addition, dual labeling of membranes and cell bodies of TFC greatly aids in tracking cellular behaviors in live embryos. For example, the dynamic formation and reorganization of follicular structures can be best appreciated in embryos co-expressing membrane-targeted mCherry and cytoplasmic or nuclear eGFP in TFC (fig. $2 \mathrm{~h}-\mathrm{j}$ ).

To fully unlock the potential of zebrafish for live imaging of thyroid growth dynamics, real-time determinations of TFC numbers should ideally be accompanied by analyses of transgenic models that allow coordinating cell cycle dynamics with specific morphogenetic processes. Live monitoring of cell cycle progression can be achieved by exploiting the Fucci (fluorescent ubiquitination-based cell cycle indicator) technology, which has recently been successfully adapted for zebrafish embryos $[37,38]$. The zebrafish Fucci system allows real-time detection of cells 
in the $\mathrm{G}_{1}$ or $\mathrm{S} / \mathrm{G}_{2} / \mathrm{M}$ phase of the cell cycle by monitoring the expression of two genetically encoded fluorescent indicators, Fucci- $\mathrm{G}_{1}$-Orange (a fusion protein of a fragment of zebrafish Cdt1 with the orange fluorescent reporter monomeric Kusabira-Orange2) and Fucci-S/G $/$ M Green (a fusion protein of a fragment of zebrafish Geminin with the green fluorescent protein monomeric Azami-Green 1) [37]. By harnessing the cell cycle-dependent ubiquitination of Cdt 1 and Geminin, the nuclei of cells in $G_{1}$ phase are labeled orange while nuclei of cells in $S / G_{2} / M$ phase are labeled in green. Although a TFC-specific Fucci model has not yet been reported, lessons learned from pancreas developmental studies [38] suggest that such a model could contribute greatly to the characterization of spatiotemporal dynamics of cell proliferation at various stages of thyroid development.

Photoconvertible fluorescent proteins (PFP) are an emerging class of fluorescent proteins that can be used for regional optical marking of individual cells or small groups of cells in order to track the fate of discrete subpopulations of tagged cells in live embryos [39]. The PFP Kaede, for example, is natively a green fluorophore but can be converted into a red fluorophore upon pulsed irradiation with near-UV light [40]. We recently generated a novel transgenic zebrafish line expressing Kaede specifically in TFC and found that global or locally restricted UV irradiation faithfully converts TFC labeling from green to red (fig. 3ae). Preliminary results from ongoing live imaging experiments with $\operatorname{tg}(\operatorname{tg}:$ Kaede) embryos indicate that photoconversion of small groups of TFC holds great potential to visualize patterns of cell movements, migration and tissue reorganization during thyroid morphogenesis [Opitz, Trubiroha and Costagliola, unpubl. data]. The future for live imaging of cellular behaviors during zebrafish thyroid organogenesis looks even brighter if one considers the expanding collection of fluorescent fusion proteins permitting novel insights into intracellular dynamics of cytoskeletal remodelling [41, 42], microtubule structures [43] or cell junctions [44]. Moreover, multi-color expression constructs have recently been developed that permit simultaneous labeling of several subcellular structures [45].

Future application of such subcellular labeling techniques in the context of thyroid morphogenesis will be greatly facilitated inasmuch as many of the aforementioned genetically encoded fluorescent probes are available as Gal4-dependent effector constructs or even as stable Gal4 effector lines. The Gal4-UAS system is a yeastderived binary expression system consisting of Gal4 activator and Gal4 effector constructs (fig. $3 \mathrm{f}$ ). Gal4 is a potent transcriptional activator that induces gene expres- sion by binding to specific DNA-binding sites called upstream activating sequences (UAS). In Gal4 activator constructs, Gal4 is expressed under the control of a tissuespecific promoter or enhancer. Gal4 effector constructs, on the other hand, contain multiple tandem UAS sites upstream of a transgene of interest. The beauty of the system is that by simple crosses of a tissue-specific Gal4 activator line with a panel of available Gal4 effector lines, Gal4-dependent expression of a variety of subcellular reporter proteins and other transgenes of interest can be efficiently realized and will occur in a tissue-specific fashion. Although an increasing number of Gal4 effector lines has been generated in the last few years, the unavailability of a thyroid-specific GAL4 activator line delayed the application of the Gal4-UAS system for studies on thyroid development. We recently generated various stable GAL4 activator lines displaying different levels of Gal4 expression in zebrafish TFC. In combination with the large number of available Gal4 effector lines, these new Gal4 driver lines are expected to open plentiful new perspectives for live imaging and the analysis of cellular dynamics during thyroid organogenesis.

In addition to tools for the visualization of cellular morphological changes, an increasing panel of signaling pathway reporter lines is now available for live monitoring of intracellular signaling activities [reviewed in 46]. These biosensor lines have been successfully used to monitor the activity of BMP, FGF, sonic hedgehog, Wnt and Notch pathways in zebrafish embryos [47-51]. Delineation of signaling pathway activities still represents a critically important task towards a better understanding of tissue-tissue interactions influencing and guiding normal thyroid organogenesis [6, 7]. Double transgenic embryos combining genetically encoded biosensor probes and fluorescent thyroid reporters therefore hold great promises to map the spatiotemporal dynamics of morphogen signaling in the region of the developing thyroid. In addition, such biosensor studies can be readily combined with chemical genetic screening approaches in order to verify the role of candidate signalling pathways for normal thyroid development [52]. Fortunately, many chemical probes known to modulate common signalling pathways in mammalian systems have been shown to faithfully function in zebrafish embryos [53, 54]. By simply adding or removing drugs at specific developmental stages from the embryo medium, chemical genetic approaches using zebrafish embryos can achieve a good temporal control of pathway modulation. Moreover, the availability of thousands of developmentally synchronized embryos allows one to scale up such screening ap- 


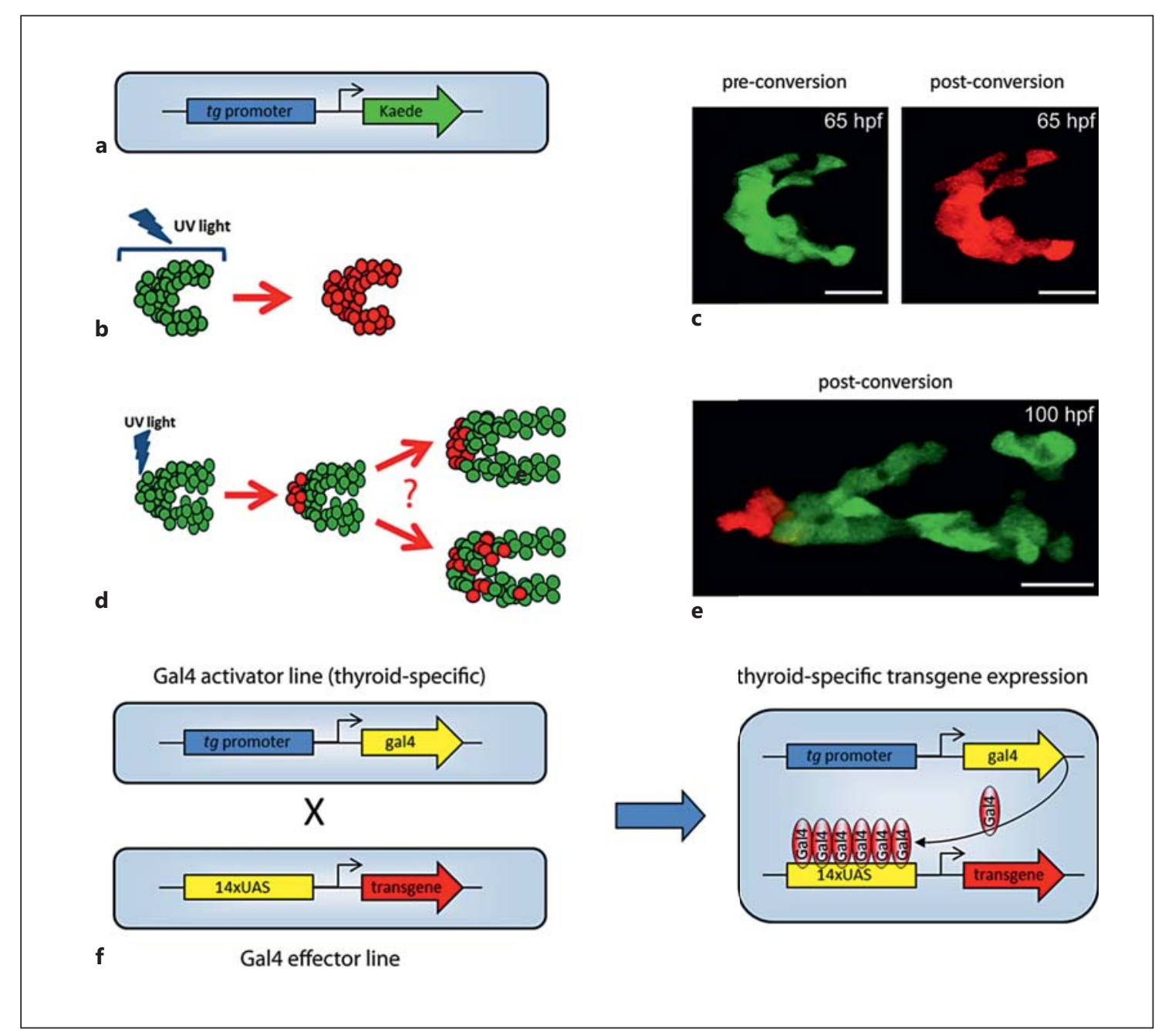

Fig. 3. a-e Transgenic embryos expressing the PFP Kaede as tools for regional optical marking of thyroid cells. a $\operatorname{tg}(\mathrm{tg}$ :Kaede) embryos express Kaede specifically in thyroid cells. b Kaede is natively a green fluorophore that is converted into a red fluorophore upon irradiation with near-UV light. c Live imaging of $\mathrm{tg}$ ( $\mathrm{tg}$ :Kaede) embryos confirmed that short UV irradiation (7-10 s) of the thyroid region faithfully converts the fluorescent labeling of all thyroid cells from green to red. Confocal projections (ventral view) are shown for the thyroid region of an embryo immediately before and after UV irradiation. Anterior is to the left. Scale: $20 \mu \mathrm{m}$. d An attractive live imaging application of this tool is to convert Kaede only in small groups of cells (e.g. by using a confocal microscope equipped with a $413 \mathrm{~nm}$ laser) and track their fate in live embryos throughout development. This potentially allows one to systematically study patterns of cell movements, migration and tissue reorganization during thyroid morphogenesis. e Live imaging of $\operatorname{tg}($ tg:Kaede) embryos demonstrating spatially restricted green-tored conversion of Kaede in a small cluster of cells at the anterior edge of the expanding thyroid. A confocal projection (ventral view) is shown for the thyroid region of a $\operatorname{tg}(\mathrm{tg}$ :Kaede) embryo immediately after spatially restricted UV irradiation. Anterior is to the left. Scale: $20 \mu \mathrm{m}$. f Schematic illustration of the binary Gal4UAS system for transgene expression. Gal4 is a yeast transcription factor that activates gene expression after binding to specific DNAbinding sites (UAS) that are not present in vertebrate genomes. Gal4 activator lines express Gal4 in a tissue-specific manner due to the use of tissue-specific promoters (e.g. the $t g$ promoter drives Gal4 expression specifically in zebrafish thyroid cells). Gal4 effector lines contain an expression cassette in which a given transgene is placed downstream of multiple tandem UAS sites and a minimal promoter. Transgene expression is only activated when Gal4 binds to the UAS sites. By crossing founder fish from activator and an effector lines, the offspring carrying both an activator and an effector allele will express the transgene in a Gal4-dependent and thus tissue-specific manner. Given the multitude of available activator (including new thyroid-specific lines) and effector lines, numerous combinatorial possibilities can be realized to direct transgene expression for studies on thyroid organogenesis. 


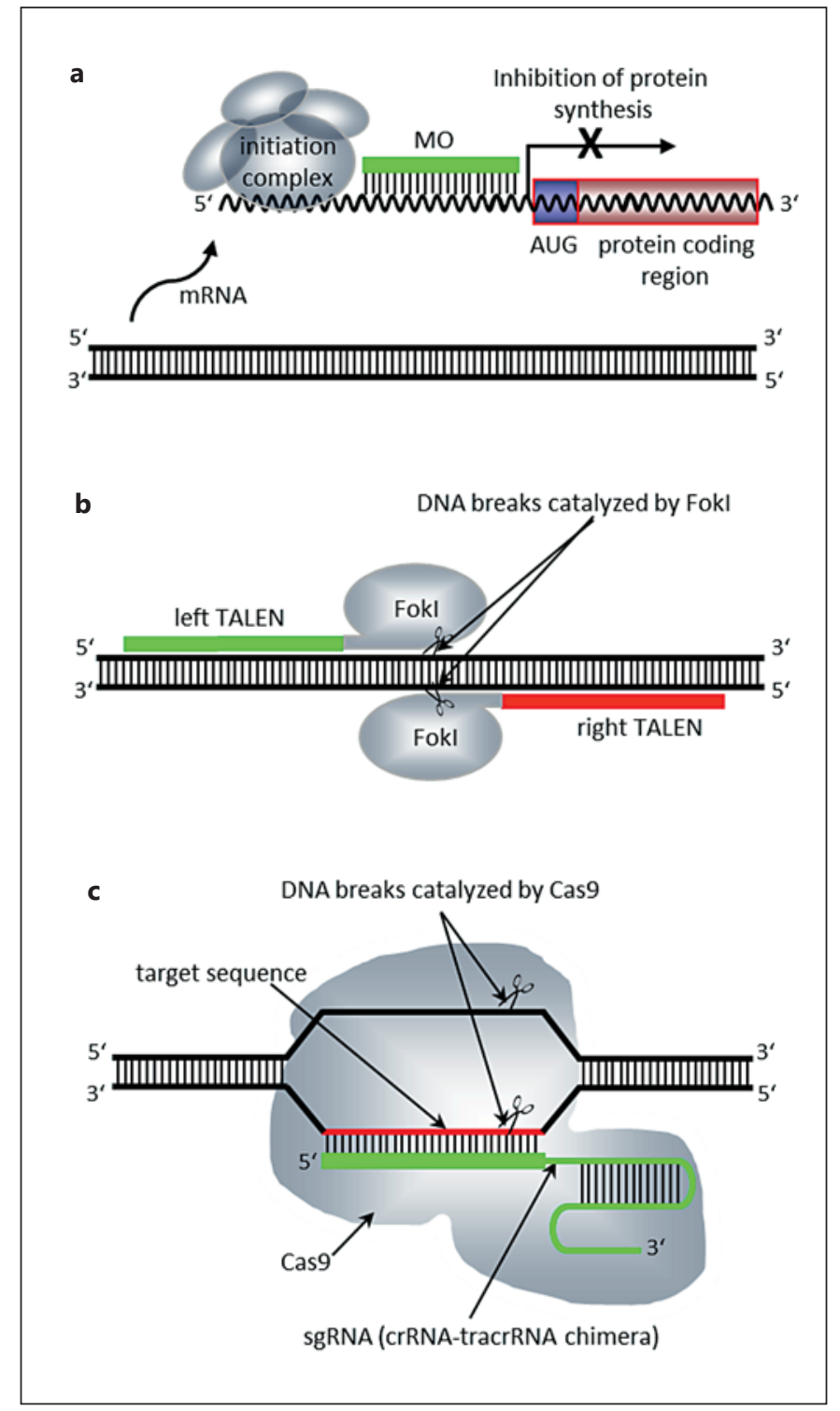

proaches. Collectively, with the availability of thyroidspecific reporter lines, the stage is now set for systematic analyses of the developmental consequences resulting from timed manipulations of common signalling pathways.

Another emerging application of transgenic reporter lines is RNA expression profiling. While the small size of the embryonic thyroid tissue precludes manual dissection, isolation of fluorescently tagged TFC from wholeembryo cell suspensions by means of fluorescence-activated cell sorting (FACS) is a vital approach to obtain thyroid-specific RNA from zebrafish embryos [Trubiroha, Opitz and Costagliola, unpubl. data]. By linking FACS
Fig. 4. LOF technologies in zebrafish. a Antisense morpholino oligonucleotides (MOs) for transient knock-down of protein function. MOs are bulk-injected into the yolk of 1- to 2-cell stage embryos. They inhibit proper mRNA translation by binding to a complimentary sequence of their target mRNA resulting in inhibition of translation initiation. Alternatively, MOs can be designed to inhibit pre-mRNA splicing or exon skipping (not shown). Thus, MOs act via interference with post-transcriptional RNA processing. Note that MOs provide only for transient effects on protein synthesis as their effective cellular concentrations decrease due to mitotic activity of the developing embryos. $\mathbf{b}$ Schematic representation of the working principle for targeted genome editing using transcription activator-like effector nucleases (TALENs). TALENs are chimeric proteins comprising a transcription activator-like effector DNA-binding domain fused to the nuclease catalytic domain of FokI. Since FokI function requires dimerization, a pair of TALENs, flanking the DNA target site, needs to be designed. Capped RNA encoding for the two TALENs is injected into 1- to 2 -cell stage embryos. In the cell nucleus, each TALEN is directed to its target sequence allowing for the dimerization of FokI, which subsequently induces a double-strand break at the selected site. Imperfect repair by the error-prone nonhomologous end-joining mechanism results in small insertions or deletions (indels). Indels that result in mutagenic frameshift changes or the introduction of an early stop codon are then selected to establish knock-out alleles. c Schematic representation of the working principle for targeted genome-editing using the CRISPR/Cas system. In contrast to the protein-based TALEN technology, the CRISPR/Cas technique is a two-component system consisting of a single guide RNA (sgRNA) and the Cas9 nuclease. The sgRNA itself is a chimeric RNA hybrid of CRISPR RNA (crRNA) and a transactivating RNA (tracrRNA). At its $5^{\prime}$ end, the sgRNA contains a 20 nucleotide long customized sequence that confers target sequence recognition via WatsonCrick base pairing with a complementary DNA sequence. The $3^{\prime}$ portion of the sgRNA is target sequence-independent and is responsible for recruiting Cas9 nuclease to the target site. The target site-specific sgRNA is co-injected with capped Cas9 RNA into 1- to 2 -cell stage embryos. In the cell nucleus, the sgRNA guides Cas9 to its target sequence where Cas 9 induces double-strand breaks. Similar to the TALEN-based approach, imperfect repair mechanisms result in small insertions or deletions (indels).

isolation of TFC with RNA amplification and RNA deep sequencing methods, exciting future perspectives of this methodological approach will include genome-wide studies of developmental changes in thyroid gene expression in zebrafish. In this respect, the availability of hundreds of developmentally synchronized embryos will provide a valuable resource for RNA isolation from embryonic thyroid cells at any desired developmental stage. From an evolutionary perspective, building this type of resource in an organism distantly related to mammals has its own value as it could enable the segregation of conserved and lineage-specific expression patterns during thyroid development in fish and mammals. 


\section{The Zebrafish Genetic Toolkit on Thyroid Organogenesis}

The modern zebrafish model is a genetically tractable experimental system that offers more than live imaging of cell behavior and morphogenic processes. It was particularly the amenability to large-scale genetic and chemical screening that propelled the rise of zebrafish as a powerful organism to interrogate gene function during developmental processes. Large zebrafish mutagenesis screens performed in the 1990s identified hundreds of genes affecting zebrafish embryonic development. Subsequent molecular and genetic analyses of mutated loci provided critical new information about endodermal organ and cardiovascular development $[19,55,56]$. One advantage of zebrafish is that such forward genetic screens can be performed at a fraction of the costs compared to those in mice. Thyroid development was not specifically addressed in any zebrafish mutagenesis screen so far but thyroid phenotypes have been identified in follow-up studies of various zebrafish mutants. For example, thyroid development is either absent or severely impaired in mutant embryos (one-eyed pinhead, casanova, bonnie and clyde, cyclops, faust) displaying defects in early endoderm formation [22]. Thyroid development is also compromised in no isthmus mutants carrying a null allele of pax $2 a$ [24] and abnormal thyroid morphogenesis has been observed in hands off (hand2) and cloche mutants both of which display defective cardiovascular development $[15,25]$. In principle, mutagenesis screens using thyroid reporter lines would represent a valuable and unbiased approach to identify novel gene activities regulating thyroid development. However, one important constraint, particularly for smaller laboratories, is that the conduct of even small-scale screens will require considerable facility space to house and maintain populations of mutagenized fish over several generations.

One major caveat of conventional gene inactivation approaches such as chemical mutagenesis is that the genetic lesion is present in all cells and at all times. Given that genes can have pleiotropic functions in multiple tissues, global gene inactivation often leads to early embryonic lethality concealing gene functions during later developmental events such as organogenesis. In mouse genetics, the introduction of site-specific recombinase systems (Cre/loxP, Flp/FRT, ФC31) as tools for genome manipulations has opened new avenues for conditional targeting of endogenous genes [57]. Until recently, techniques similar to homologous recombination in mouse embryonic stem cells for the generation of conditional alleles were not available for zebrafish. What is possible in zebrafish, how-

An Update on the Zebrafish Toolbox ever, is to use transgenesis for genomic insertion of exogenous DNA sequences that are flanked by recombinase recognition sites. Once integrated, Cre and Flp recombinases were shown to faithfully carry out excision, inversion or exchange of such DNA sequences [58-60]. To date, the Cre/loxP system has been used in zebrafish predominantly for lineage-tracing experiments [61-63] and to control transgene expression in a tissue- and time-dependent manner [64]. One exciting recent development is the application of site-specific recombinase systems in conjunction with gene-trapping approaches. A new generation of conditional gene-trap systems permits not only the fluorescent tagging of trapped proteins and their quantitative removal, but also offers the advantage of conditional inactivation or rescue of trapped proteins [6567]. The value of these advanced gene-trap models has clearly been demonstrated for analyses of spatiotemporal dynamics of protein expression, cellular behaviors and mutant phenotypic traits [67]. Although conditional gene-trap models have yet to be established for thyroidspecific genes, the rapidly expanding collection of conditional mutant lines (http://zfishbook.org/, http://www. fliptrap.org) will hopefully become an important resource also for zebrafish thyroid research $[66,68]$. In summary, a series of technological advancements in the recent past have made zebrafish an ideal model organism to study the basic mechanisms underlying organ development, and application of these novel techniques and methods in the context of thyroid morphogenesis is expected to provide for new and innovative research strategies to enhance the understanding of vertebrate thyroid development.

\section{Perspectives for Modeling Human Thyroid Diseases in Zebrafish}

Along with the introduction of novel molecular and genetic technologies, zebrafish also became an important model for several human diseases [19-21]. The question thus arises on how zebrafish could aid in modeling human thyroid diseases, in particular congenital thyroid diseases resulting from defects in embryonic and fetal thyroid development. At present, one of the major problems in understanding congenital thyroid defects is the lack of knowledge on the pathogenic gene variants responsible for abnormal thyroid development in the human embryo and fetus. As already shown for various human diseases, cutting-edge technologies based on nextgenerationsequencing techniques (e.g. exome sequencing, epigenome analyses and RNA sequencing) allow now un- 
precedented in-depth analyses of patient samples [69, 70]. Undoubtedly, application of these techniques to cohorts of $\mathrm{CH}$ patients will accelerate the identification of new candidate genetic causes for congenital thyroid diseases. The challenge that will come along with this flood of novel genetic information is to identify powerful systems for functional validation of putatively pathogenic gene variants. Stem cell-based models in conjunction with mouse genetic models will certainly play a vital role in such functional validation strategies $[3,71]$. However, certain salient features of zebrafish embryos can make this system a preferable experimental tool to dissect the functional role for a subset of these candidate genes. Central for its utility as a model for human diseases are techniques that permit to readily generate zebrafish models to perform loss-of-function (LOF) and gain-of-function (GOF) studies for a given candidate gene.

In the past, the unavailability of efficient homologous recombination and RNAi techniques for zebrafish LOF studies propelled the widespread use of antisense morpholino oligonucleotides (MOs) for phenocopy experiments in zebrafish embryos [72]. MOs are nonionic DNA analogs composed of a phosphorodiamidate backbone with a morpholine ring in lieu of a ribose ring. These structural features make them resistant to digestion by nucleases and therefore remarkably stable. The working principle of MOs is binding to a complimentary sequence in their target RNA resulting in inhibition of translation initiation or aberrant splicing (fig. 4a). Thus, MO-mediated gene knock-down is not based on genetic manipulations but on $\mathrm{MO}$ interference with post-transcriptional RNA processing. In combination with the near complete sequencing and assembly of the zebrafish genome, it was particularly the ease of MO injections into the yolk of 1to 2-cell stage embryos that boosted the use of MOs as a phenocopy strategy to study mutant phenotypes. It is worth noting that MOs have also been successfully used to suppress microRNA and lnRNA function in zebrafish $[73,74]$. MO-mediated gene knock-down has successfully been used to delineate the role of various genes during zebrafish thyroid organogenesis including $n k x 2.1 a$ [22], hhex [22], tshr [26], vegfa [14], jagla [75], jag1b [75], bcl2l [76] and ltbp3 [15]. Notably, MO-mediated knock-down of $n k x 2.1 a$ [22] and $t s h r$ [26] function in zebrafish embryos recapitulated thyroid phenotypes observed in $\mathrm{CH}$ patients and mouse models with inactivating mutations of NKX2.1 (hypoplasia, athyreosis) and TSHR (hypofunctional gland with reduced number of follicles) [1-6]. These data clearly strengthen the view of zebrafish as a valuable system to model congenital thyroid diseases.
The use of MOs offers several advantages for phenocopy studies. Co-injection of different MOs, or MO injection into mutant embryos, has proven a vital approach to reveal redundant and combinatorial functions of developmental genes [77, 78]. If carefully titrated, co-injection of different MOs at submaximal doses can also help to address the question whether multiple genes interact genetically in a given process [79]. Moreover, analyses of LOF phenotypes in multiple organs and tissues is greatly facilitated by injecting the same MO into a panel of tissue-specific reporter embryos [15]. On the flipside, it is essential to realize that, as with any antisense experiment, rigorous controls are critical to interpreting $\mathrm{MO}$-induced phenotypes and to distinguish off-target effects from phenotypic changes due to the specific knock-down of the gene of interest $[72,80]$. One also has to consider that the MO technology provides only for transient effects on protein synthesis. Because MOs are bulk-injected into embryos at the 1- to 2-cell stage, the effective cellular MO concentrations decrease due to mitotic activity of the developing embryos. Conservative estimates suggest that proper MO efficacy is limited to the first 3-4 days of development [72].

While the application of MOs has been for years the most common approach to generate LOF models in zebrafish, the recent development of custom-designed nucleases revolutionized the way LOF and other genetic models can be generated in zebrafish. Designed nucleases such as zinc-finger nucleases (ZFNs) or transcription activatorlike effector nucleases (TALENs) permit precise and targeted modifications of the zebrafish genome [81-84]. Both types of nucleases share a common working principle for genome editing. A DNA-binding moiety, specific for the genomic DNA sequence to be targeted, is coupled to the nonspecific FokI cleavage domain and introduction of a pair of such fusion constructs into the nucleus results in DNA binding, dimerization of FokI and generation of double-stranded DNA breaks at the selected site (fig. 4b). Imperfect repair by the error-prone nonhomologous end joining mechanism results in small insertions or deletions that cause mutagenic frameshift changes. The ZFN technology has spread only slowly in the zebrafish field as the design of effective yet specific ZFN reagents proved a difficult and laborious task in many instances. The more recently introduced TALEN technology overcomes several of the limitations of ZFN [83, 85]. Specifically, TALENs have a more simple code for DNA site recognition facilitating streamlined design and assembly of functional TALEN reagents in individual laboratories. In addition, recent studies indicated that TALENs produce minimal off-target effects and are more mutagenic than ZFN [86-89]. Our 
own ongoing studies on TALEN-mediated genome editing of zebrafish loci relevant for thyroid organogenesis (e.g. $n k x 2.1$ la locus on chromosome 20) confirmed these findings and revealed impressive mutagenic efficiencies with more than $90 \%$ of injected embryos bearing mutations at the target site many of which turned out to be biallelic mutations [Opitz and Costagliola, unpubl. data].

In early 2013, a new class of tools was introduced for the precise and efficient genome editing in zebrafish, the type II prokaryotic clustered regularly interspaced short palindromic repeats (CRISPR)/CRISPR-associated proteins (Cas) system [90]. The major advantage offered by the CRISPR/Cas technique relative to the protein-based TALEN technology is the relative ease of assembling the RNA-guided Cas nuclease system. The CRISPR/Cas system merely requires in vitro synthesis of a target site-specific single guide RNA (sgRNA) that, after co-injection with capped Cas9 RNA, will guide the Cas nuclease complex to introduce site-specific DNA double-stranded breaks (fig. 4c). Meanwhile, the CRISPR/Cas technology has proven in several studies to be highly efficient in modifying various genomic loci in zebrafish with efficiencies comparable to the TALEN technology [90-92]. Importantly, efficient germline transmission has been demonstrated for genomic modifications introduced by either TALEN or CRISPR/Cas systems $[82,83,91,92]$. Another step-change in genome-editing perspectives in zebrafish was the demonstration of homology-directed repair by co-delivering donor DNA partially homologous to the genomic target site together with TALEN pairs or with sgRNA/Cas9 [84, 89, 92].

To date, there are no published studies addressing thyroid development in zebrafish mutants generated by any of these novel genome-editing tools. Nevertheless, from the data available on other organ systems, it is already clear that these recent technological developments will have a tremendous impact on the utility of the zebrafish system for the modeling of human thyroid diseases. First, the ease of delivering the genome-editing reagents into early zebrafish embryos by microinjection and the efficiency of TALEN and CRISPR/Cas systems to induce heritable targeted gene disruption allow for the rapid generation of many new zebrafish mutant models for genes implicated in or associated with human congenital thyroid defects. Second, the demonstration of precise template-directed genome modification in zebrafish including precise single nucleotide mutations, introduction of loxP or FRT sites and knock-in of large DNA fragments provides the zebrafish researcher now for the first time with a reverse genetics toolkit that is comparable to mouse genetics. With such powerful tools becoming available, the zebrafish system is expected to contribute new and innovative experimental models for advanced functional genomic studies on thyroid development. In particular, the combination of available reporter tools with the opportunity to rapidly design and generate genetic LOF models à la carte will greatly enhance the amenability of zebrafish for larger-scale in vivo evaluation of candidate genes for human thyroid abnormalities.

\section{Conclusion and Perspectives}

Zebrafish embryos lend themselves particularly well to live imaging studies of thyroid organogenesis due to their small size, rapid development and optical transparency. Given the conservation of molecular mechanisms regulating thyroid development in zebrafish and mammalian model species, zebrafish embryos will provide a valid and powerful model to decipher the dynamics of cellular behavior during distinct morphogenetic processes. The availability of transgenic reporter tools is a key factor paving the way for novel and innovative experimental approaches including real-time in vivo monitoring of thyroid tissue growth kinetics, cell migration, tissue reorganization and follicle formation. FACS isolation of fluorescent thyroid cells from embryos at different developmental stages opens the possibility for studying the dynamics of the TFC transcriptome during development. In addition, combining the use of signaling pathway reporters with chemical genetic screens is expected to rapidly yield new insights into the role of extrinsic signaling cues for thyroid morphogenesis. To fully unlock the potential of zebrafish, however, more efforts are still needed to develop novel transgenic tools. At present, the very early steps of thyroid development cannot yet be addressed with the available transgenic tools. Therefore, one important task will be the identification of regulatory DNA regions that drive robust transgene expression in cells of the early thyroid anlage or in endodermal precursor cells. Since the cis-regulatory landscape controlling the expression of key thyroid transcription factors such as $n k \times 2.1 a$ or pax $2 a$ is very complex and likely involves the interplay between distinct enhancer and promoter sequences, the development of early active reporter tools will probably require the use of bacterial artificial chromosomes (BACs) for transgenesis [93] or the generation of knock-in models using new genome-editing techniques [89].

Next-generation sequencing technologies have recently revolutionized the search for disease-causing genes [69, 
70] and the next few years will likely experience an increasing use of these information-rich sequencing technologies to identify novel putatively pathogenic gene mutations in TD patients. The ready generation of corresponding genetic zebrafish models will be a prerequisite to efficiently harness the specific advantages of zebrafish embryos for a phenotypic characterization of novel TD-causing candidate genes. Therefore, we consider the broad application of custom-designed nucleases for targeted genome editing as an important research topic in order to propel the use of zebrafish embryos for the modeling of human thyroid diseases. In this respect, it will be paramount to rapidly develop protocols that not only allow for the generation of small mutagenic lesions, but also for the generation of conditional alleles of putative candidate genes.

\section{Acknowledgements}

We thank the members of the Costagliola lab for fruitful discussions and comments on the manuscript. The Costagliola lab is supported by the Belgian Fonds de la Recherche Scientifique Médicale (FRSM [3]3_4598_12), by Action de Recherche Concertée de la Communauté Française de Belgique (ARC No. AUWB2012-12/17-UMONS/ULB), by Belgian Televie, by Fonds d'Encouragement à la Recherche (ULB) and grants from the Belgian National Fund for Scientific Research (FNRS). R.O. is FNRS Postdoctoral Researcher, F.A. is FRIA research fellows, and S.C. is FNRS Senior Research Associate.

\section{Disclosure Statement}

The authors have no conflicts of interest to disclose.

\section{References}

1 Rastogi MV, LaFranchi SH: Congenital hypothyroidism. Orphanet J Rare Dis 2010;10:517.

2 Pohlenz J, van Vliet G: Developmental abnormalities of the thyroid; in Weiss RE, Refetoff $S$ (eds): Genetic Diagnosis of Endocrine Disorders. London, Elsevier, 2010, pp 97-104.

3 De Felice M, Di Lauro R: Thyroid development and its disorders: genetics and molecular mechanisms. Endocr Rev 2004;25:722746.

4 Castanet M, Marinovic D, Polak M, Léger J: Epidemiology of thyroid dysgenesis: the familial component. Horm Res Paediatr 2010; 73:231-237.

5 Polak M, Sura-Trueba S, Chauty A, Szinnai G, Carré A, Castanet M: Molecular mechanisms of thyroid dysgenesis. Horm Res 2004;62:1421.

6 6 De Felice M, Di Lauro R: Minireview: intrinsic and extrinsic factors in thyroid gland development: an update. Endocrinology 2011; 152:2948-2956.

7 Fagman H, Nilsson M: Morphogenetics of early thyroid development. J Mol Endocrinol 2011;46:R33-R42.

8 Kimura S, Ward JM, Minoo P: Thyroid-specific enhancer-binding protein/thyroid transcription factor 1 is not required for the initial specification of the thyroid and lung primordia. Biochimie 1999;81:321-327.

-9 Mansouri A, Chowdhury K, Gruss P: Follicular cells of the thyroid gland require Pax8 gene function. Nat Genet 1998;19:87-90.

$\checkmark 10$ De Felice M, Ovitt C, Biffali E, RodriguezMallon A, Arra C, Anastassiadis K, Macchia PE, Mattei MG, Mariano A, Schöler H, Macchia V, Di Lauro R: A mouse model for hereditary thyroid dysgenesis and cleft palate. Nat Genet 1998;19:395-398.
Parlato R, Rosica A, Rodriguez-Mallon A, Affuso A, Postiglione MP, Arra C, Mansouri A, Kimura S, Di Lauro R, De Felice M: An integrated regulatory network controlling survival and migration in thyroid organogenesis. Dev Biol 2004;276:464-475.

12 Deladoëy J, Vassart G, Van Vliet G: Possible non-mendelian mechanisms of thyroid dysgenesis. Endocr Dev 2007;10:29-42.

13 Fagman H, Grände M, Gritli-Linde A, Nilsson M: Genetic deletion of sonic hedgehog causes hemiagenesis and ectopic development of the thyroid in mouse. Am J Pathol 2004; 164:1865-1872.

14 Alt B, Elsalini OA, Schrumpf P, Haufs N, Lawson ND, Schwabe GC, Mundlos S, Grüters A, Krude H, Rohr KB: Arteries define the position of the thyroid gland during its developmental relocalisation. Development 2006; 133:3797-3804

15 Opitz R, Maquet E, Huisken J, Antonica F, Trubiroha A, Pottier G, Janssens V, Costagliola S: Transgenic zebrafish illuminate the dynamics of thyroid morphogenesis and its relationship to cardiovascular development. Dev Biol 2012;372:203-216.

16 Thisse C, Zon LI: Organogenesis - heart and blood formation from the zebrafish point of view. Science 2002;295:457-462.

17 Stuckenholz C, Ulanch PE, Bahary N: From guts to brains: using zebrafish genetics to understand the innards of organogenesis. Curr Top Dev Biol 2005;65:47-82.

18 Swanhart LM, Cosentino CC, Diep CQ, Davidson AJ, de Caestecker M, Hukriede NA: Zebrafish kidney development: basic science to translational research. Birth Defects Res C Embryo Today 2011;93:141-156.

19 Bakkers J: Zebrafish as a model to study cardiac development and human cardiac disease. Cardiovasc Res 2011;91:279-288.
20 Jing L, Zon LI: Zebrafish as a model for normal and malignant hematopoiesis. Dis Model Mech 2011;4:433-438.

21 Löhr H, Hammerschmidt M: Zebrafish in endocrine systems: recent advances and implications for human disease. Annu Rev Physiol 2011;73:183-211.

22 Elsalini OA, von Gartzen J, Cramer M, Rohr KB: Zebrafish hhex, nk2.1a, and pax2.1 regulate thyroid growth and differentiation downstream of nodal-dependent transcription factors. Dev Biol 2003;263:67-80.

-23 Alt B, Reibe S, Feitosa NM, Elsalini OA, Wendl T, Rohr KB: Analysis of origin and growth of the thyroid gland in zebrafish. Dev Dyn 2006;235:1872-1883.

24 Wendl T, Lun K, Mione M, Favor J, Brand M, Wilson SW, Rohr KB: Pax2.1 is required for the development of thyroid follicles in zebrafish. Development 2002;129:3751-3760.

25 Wendl T, Adzic D, Schoenebeck JJ, Scholpp S, Brand M, Yelon D, Rohr KB: Early developmental specification of the thyroid gland depends on Han-expressing surrounding tissue and on FGF signals. Development 2007;134: 2871-2879.

26 Opitz R, Maquet E, Zoenen M, Dadhich R, Costagliola S: TSH receptor function is required for normal thyroid differentiation in zebrafish. Mol Endocrinol 2011;25:15791599.

27 Porazzi P, Calebiro D, Benato F, Tiso N, Persani L: Thyroid gland development and function in the zebrafish model. Mol Cell Endocrinol 2009;312:14-23.

28 Fagman H, Nilsson M: Morphogenesis of the thyroid gland. Mol Cell Endocrinol 2010;323: 35-54.

29 Kawakami K: Transposon tools and methods in zebrafish. Dev Dyn 2005;234:244-254. 
30 Villefranc JA, Amigo J, Lawson ND: Gateway compatible vectors for analysis of gene function in the zebrafish. Dev Dyn 2007;236: 3077-3087.

- 31 Kwan KM, Fujimoto E, Grabher C, Mangum BD, Hardy ME, Campbell DS, Parant JM, Yost HJ, Kanki JP, Chien CB: The Tol2kit: a multisite gateway-based construction kit for Tol2 transposon transgenesis constructs. Dev Dyn 2007;236:3088-3099.

- 32 Bussmann J, Schulte-Merker S: Rapid BAC selection for tol2-mediated transgenesis in zebrafish. Development 2011;138:4327-4332.

33 Kamei M, Weinstein BM: Long-term timelapse fluorescence imaging of developing zebrafish. Zebrafish 2005;2:113-123.

34 Weber M, Huisken J: Light sheet microscopy for real-time developmental biology. Curr Opin Genet Dev 2011;21:566-572.

- 35 Kaufmann A, Mickoleit M, Weber M, Huisken J: Multilayer mounting enables long-term imaging of zebrafish development in a light sheet microscope. Development 2012;139: 3242-3247.

36 Huisken J: Slicing embryos gently with laser light sheets. Bioessays 2012;34:406-411.

- 37 Sugiyama M, Sakaue-Sawano A, Iimura T, Fukami K, Kitaguchi T, Kawakami K, Okamoto H, Higashijima S, Miyawaki A: Illuminating cell-cycle progression in the developing zebrafish embryo. Proc Natl Acad Sci USA 2009; 106:20812-20817.

- 38 Ninov N, Hesselson D, Gut P, Zhou A, Fidelin K, Stainier DY: Metabolic regulation of cellular plasticity in the pancreas. Curr Biol 2013; 23:1242-1250.

- 39 Hatta K, Tsujii H, Omura T: Cell tracking using a photoconvertible fluorescent protein. Nat Protoc 2006;1:960-967.

-40 Ando R, Hama H, Yamamoto-Hino M, Mizuno H, Miyawaki A: An optical marker based on the UV-induced green-to-red photoconversion of a fluorescent protein. Proc Natl Acad Sci USA 2002;99:12651-12656.

-41 Riedl J, Crevenna AH, Kessenbrock K, Yu JH, Neukirchen D, Bista M, Bradke F, Jenne D, Holak TA, Werb Z, Sixt M, WedlichSoldner R: Lifeact: a versatile marker to visualize F-actin. Nat Methods 2008;5:605607.

- 42 Seiler C, Davuluri G, Abrams J, Byfield FJ, Janmey PA, Pack M: Smooth muscle tension induces invasive remodeling of the zebrafish intestine. PLoS Biol 2012;10:e1001386.

- 43 Asakawa K, Kawakami K: A transgenic zebrafish for monitoring in vivo microtubule structures. Dev Dyn 2010;239:2695-2699.

-44 Herwig L, Blum Y, Krudewig A, Ellertsdottir E, Lenard A, Belting HG, Affolter M: Distinct cellular mechanisms of blood vessel fusion in the zebrafish embryo. Curr Biol 2011;21: 1942-1948.

-45 Distel M, Hocking JC, Volkmann K, Köster RW: The centrosome neither persistently leads migration nor determines the site of axonogenesis in migrating neurons in vivo. J Cell Biol 2010;191:875-890.
46 Moro E, Vettori A, Porazzi P, Schiavone M, Rampazzo E, Casari A, Ek O, Facchinello N, Astone M, Zancan I, Milanetto M, Tiso N, Argenton F: Generation and application of signaling pathway reporter lines in zebrafish. Mol Genet Genomics 2013;288:231-242.

47 Laux DW, Febbo JA, Roman BL: Dynamic analysis of BMP-responsive smad activity in live zebrafish embryos. Dev Dyn 2011;240: 682-694.

48 Molina GA, Watkins SC, Tsang M: Generation of FGF reporter transgenic zebrafish and their utility in chemical screens. BMC Dev Biol 2007;7:62.

49 Choi WY, Gemberling M, Wang J, Holdway JE, Shen MC, Karlstrom RO, Poss KD: In vivo monitoring of cardiomyocyte proliferation to identify chemical modifiers of heart regeneration. Development 2013;140:660-666.

50 Moro E, Ozhan-Kizil G, Mongera A, Beis D, Wierzbicki C, Young RM, Bournele D, Domenichini A, Valdivia LE, Lum L, Chen C, Amatruda JF, Tiso N, Weidinger G, Argenton F: In vivo Wnt signaling tracing through a transgenic biosensor fish reveals novel activity domains. Dev Biol 2012;366:327-340.

51 Ninov N, Borius M, Stainier DY: Different levels of Notch signaling regulate quiescence, renewal and differentiation in pancreatic endocrine progenitors. Development 2012;139: 1557-1567.

52 Opitz R, Vandernoot I, Haerlingen B, Trubiroha A, Costagliola S: Small molecule screening identifies signaling pathways regulating thyroid morphogenesis in zebrafish embryos (abstract). Eur Thyroid J 2013;2(suppl 1):88.

53 Peterson RT, Link BA, Dowling JE, Schreiber SL: Small molecule developmental screens reveal the logic and timing of vertebrate development. Proc Natl Acad Sci USA 2000;97: 12965-12969.

54 Tamplin OJ, White RM, Jing L, Kaufman CK, Lacadie SA, Li P, Taylor AM, Zon LI: Small molecule screening in zebrafish: swimming in potential drug therapies. Wiley Interdiscip Rev Dev Biol 2012;1:459-468.

55 Stainier DY: Zebrafish genetics and vertebrate heart formation. Nat Rev Genet 2001;2:39-48.

56 Ober EA, Verkade H, Field HA, Stainier DY: Mesodermal Wnt $2 \mathrm{~b}$ signalling positively regulates liver specification. Nature 2006;442: 688-691.

57 Branda CS, Dymecki SM: Talking about a revolution: The impact of site-specific recombinases on genetic analyses in mice. Dev Cell 2004;6:7-28.

58 Hans S, Freudenreich D, Geffarth M, Kaslin J, Machate A, Brand M: Generation of a nonleaky heat shock-inducible Cre line for conditional Cre/lox strategies in zebrafish. Dev Dyn 2011;240:108-115.

59 Boniface EJ, Lu J, Victoroff T, Zhu M, Chen W: FlEx-based transgenic reporter lines for visualization of Cre and Flp activity in live zebrafish. Genesis 2009;47:484-491.
60 Hu G, Goll MG, Fisher S: ФC31 integrase mediates efficient cassette exchange in the zebrafish germline. Dev Dyn 2011;240:2101-2107.

61 Mongera A, Singh AP, Levesque MP, Chen YY, Konstantinidis P, Nüsslein-Volhard C: Genetic lineage labeling in zebrafish uncovers novel neural crest contributions to the head, including gill pillar cells. Development 2013. 140:916-925

62 Zhou Y, Cashman TJ, Nevis KR, Obregon P, Carney SA, Liu Y, Gu A, Mosimann C, Sondalle S, Peterson RE, Heideman W, Burns CE, Burns CG: Latent TGF- $\beta$ binding protein 3 identifies a second heart field in zebrafish. $\mathrm{Na}$ ture 2011;474:645-648.

63 Kikuchi K, Holdway JE, Werdich AA, Anderson RM, Fang Y, Egnaczyk GF, Evans T, Macrae CA, Stainier DY, Poss KD: Primary contribution to zebrafish heart regeneration by gata4[+] cardiomyocytes. Nature 2010;464: 601-605.

64 Hesselson D, Anderson RM, Beinat M, Stainier DY: Distinct populations of quiescent and proliferative pancreatic $\beta$-cells identified by HOTcre-mediated labeling. Proc Natl Acad Sci USA 2009;106:14896-14901.

65 Clark KJ, Balciunas D, Pogoda HM, Ding Y, Westcot SE, Bedell VM, Greenwood TM, Urban MD, Skuster KJ, Petzold AM, Ni J, Nielsen AL, Patowary A, Scaria V, Sivasubbu S, Xu X, Hammerschmidt M, Ekker SC: In vivo protein trapping produces a functional expression codex of the vertebrate proteome. Nat Methods 2011;8:506-515.

-66 Trinh le A, Hochgreb T, Graham M, Wu D, Ruf-Zamojski F, Jayasena CS, Saxena A, Hawk R, Gonzalez-Serricchio A, Dixson A, Chow E, Gonzales C, Leung HY, Solomon I, Bronner-Fraser M, Megason SG, Fraser SE: A versatile gene trap to visualize and interrogate the function of the vertebrate proteome. Genes Dev 2011;25:2306-2320.

67 Ni TT, Lu J, Zhu M, Maddison LA, Boyd KL, Huskey L, Ju B, Hesselson D, Zhong TP, PageMcCaw PS, Stainier DY, Chen W: Conditional control of gene function by an invertible gene trap in zebrafish. Proc Natl Acad Sci USA 2012;109:15389-15394.

68 Ding Y, Liu W, Deng Y, Jomok B, Yang J, Huang W, Clark KJ, Zhong TP, Lin X, Ekker SC, Xu X: Trapping cardiac recessive mutants via expression-based insertional mutagenesis screening. Circ Res 2013;112:606-617.

69 Metzker ML: Sequencing technologies - the next generation. Nat Rev Genet 2010;11:3146.

70 Rabbani B, Mahdieh N, Hosomichi K, Nakaoka H, Inoue I: Next-generation sequencing: impact of exome sequencing in characterizing mendelian disorders. J Hum Genet 2012;57: 621-632.

71 Antonica F, Kasprzyk DF, Opitz R, Iacovino M, Liao XH, Dumitrescu AM, Refetoff S, Peremans K, Manto M, Kyba M, Costagliola $S$ : Generation of functional thyroid from embryonic stem cells. Nature 2012;491:66-71. 
72 Eisen JS, Smith JC: Controlling morpholino experiments: don't stop making antisense. Development 2008; 135:1735-1743.

-73 Kloosterman WP, Lagendijk AK, Ketting RF, Moulton JD, Plasterk RH: Targeted inhibition of miRNA maturation with morpholinos reveals a role for miR-375 in pancreatic islet development. PLoS Biol 2007;5:e203.

74 Choi WY, Giraldez AJ, Schier AF: Target protectors reveal dampening and balancing of nodal agonist and antagonist by miR-430. Science 2007;318:271-274.

75 Porazzi P, Marelli F, Benato F, de Filippis T, Calebiro D, Argenton F, Tiso N, Persani L: Disruptions of global and JAGGED1-mediated notch signaling affect thyroid morphogenesis in the zebrafish. Endocrinology 2012;153: 5645-5658.

-76 Porreca I, De Felice E, Fagman H, Di Lauro R, Sordino P: Zebrafish bcl2l is a survival factor in thyroid development. Dev Biol 2012;366: 142-152.

-77 Maroon H, Walshe J, Mahmood R, Kiefer P, Dickson C, Mason I: Fgf3 and Fgf8 are required together for formation of the otic placode and vesicle. Development 2002;129: 2099-2108.

78 Pham VN, Lawson ND, Mugford JW, Dye L, Castranova D, Lo B, Weinstein BM: Combinatorial function of ETS transcription factors in the developing vasculature. Dev Biol 2007; 303:772-783.

-79 Stalmans I, Lambrechts D, De Smet F, Jansen S, Wang J, Maity S, Kneer P, von der Ohe M, Swillen A, Maes C, Gewillig M, Molin DG, Hellings P, Boetel T, Haardt M, Compernolle V, Dewerchin M, Plaisance S, Vlietinck R, Emanuel B, Gittenberger-de Groot AC, Scam- bler P, Morrow B, Driscol DA, Moons L, Esguerra CV, Carmeliet G, Behn-Krappa A, Devriendt K, Collen D, Conway SJ, Carmeliet P: VEGF: a modifier of the del22q11 (DiGeorge) syndrome? Nat Med 2003;9:173-182.

80 Robu ME, Larson JD, Nasevicius A, Beiraghi S, Brenner C, Farber SA, Ekker SC: p53 activation by knockdown technologies. PLoS Genet 2007;3:e78.

81 Doyon Y, McCammon JM, Miller JC, Faraji F, Ngo C, Katibah GE, Amora R, Hocking TD, Zhang L, Rebar EJ, Gregory PD, Urnov FD, Amacher SL: Heritable targeted gene disruption in zebrafish using designed zinc-finger nucleases. Nat Biotechnol 2008;26:702-708.

82 Meng X, Noyes MB, Zhu LJ, Lawson ND, Wolfe SA: Targeted gene inactivation in zebrafish using engineered zinc-finger nucleases. Nat Biotechnol 2008;26:695-701.

83 Huang P, Xiao A, Zhou M, Zhu Z, Lin S, Zhang B: Heritable gene targeting in zebrafish using customized TALENs. Nat Biotechnol 2011;29:699-700.

84 Bedell VM, Wang Y, Campbell JM, Poshusta TL, Starker CG, Krug RG 2nd, Tan W, Penheiter SG, Ma AC, Leung AY, Fahrenkrug SC, Carlson DF, Voytas DF, Clark KJ, Essner JJ, Ekker SC: In vivo genome editing using a high-efficiency TALEN system. Nature 2012; 491:114-118.

85 Sander JD, Cade L, Khayter C, Reyon D, Peterson RT, Joung JK, Yeh JR: Targeted gene disruption in somatic zebrafish cells using engineered TALENs. Nat Biotechnol 2011;29: 697-698.

86 Chen S, Oikonomou G, Chiu CN, Niles BJ, Liu J, Lee DA, Antoshechkin I, Prober DA: A large-scale in vivo analysis reveals that
TALENs are significantly more mutagenic than ZFNs generated using context-dependent assembly. Nucleic Acids Res 2013;41: 2769-2778.

87 Dahlem TJ, Hoshijima K, Jurynec MJ, Gunther D, Starker CG, Locke AS, Weis AM, Voytas DF, Grunwald DJ: Simple methods for generating and detecting locus-specific mutations induced with TALENs in the zebrafish genome. PLoS Genet 2012;8:e1002861.

88 Cade L, Reyon D, Hwang WY, Tsai SQ, Patel S, Khayter C, Joung JK, Sander JD, Peterson RT, Yeh JR: Highly efficient generation of heritable zebrafish gene mutations using homo- and heterodimeric TALENs. Nucleic Acids Res 2012;40:8001-8010.

89 Zu Y, Tong X, Wang Z, Liu D, Pan R, Li Z, Hu Y, Luo Z, Huang P, Wu Q, Zhu Z, Zhang B, Lin S: TALEN-mediated precise genome modification by homologous recombination in zebrafish. Nat Methods 2013;10:329-331.

90 Hwang WY, Fu Y, Reyon D, Maeder ML, Tsai SQ, Sander JD, Peterson RT, Yeh JR, Joung JK: Efficient genome editing in zebrafish using a CRISPR-Cas system. Nat Biotechnol 2013;31:227-229.

91 Hwang WY, Fu Y, Reyon D, Maeder ML, Kaini P, Sander JD, Joung JK, Peterson RT, Yeh JR: Heritable and precise zebrafish genome editing using a CRISPR-Cas system. PLoS One 2013;8:e68708.

92 Jao LE, Wente SR, Chen W: Efficient multiplex biallelic zebrafish genome editing using a CRISPR nuclease system. Proc Natl Acad Sci USA 2013;110:13904-13909.

93 Suster ML, Abe G, Schouw A, Kawakami K: Transposon-mediated BAC transgenesis in zebrafish. Nat Protoc 2011;6:1998-2021. 